\title{
Combination of FLG mutations and SNPs of IL-17A and IL-19 influence on atopic dermatitis occurrence
}

\author{
Jolanta Klonowska1, Jolanta Gleń², Roman J. Nowicki², Magdalena Trzeciak² \\ ${ }^{1}$ Independent Public Health Care Unit of Rypin, Rypin, Poland \\ ${ }^{2}$ Department of Dermatology, Venereology and Allergology, Medical University of Gdansk, Gdansk, Poland \\ Adv Dermatol Allergol 2022; XXXIX (1): 200-208 \\ DOI: https://doi.org/10.5114/ada.2021.105412
}

\begin{abstract}
Introduction: Atopic dermatitis (AD) is a heterogeneous inflammatory skin disease. A fresh look on the AD pathophysiology has focused on the skin barrier defect and immune dysfunctions. IL-17A and IL-19 seem to play role in AD pathogenesis.

Aim: The aim was to investigate associations of SNPs of IL-17A (rs2275913) and IL-19 (rs22431188) with AD features, course and occurrence. Searching for prognostic panels composed of FLG (2282del4, R501X) mutations with IL-17A and IL-19 polymorphisms.

Material and methods: Blood samples were collected from 239 patients with AD and 170 controls. Two SNPs, IL-17A and IL-19 and FLG null mutations were analyzed. PCR and RFLP restriction fragment length polymorphism analysis were used. SCORAD score to establish AD severity, VAS to estimate pruritus.

Results: None polymorphisms of studied cytokines caused more frequent AD occurrence compared to controls. We found no associations between IL-17A and IL-19 gene polymorphisms and AD severity (respectively $p=0.954$; $p=0.498)$, IgE level $(p=0.707 ; p=0.584)$, VAS $(p=0.953 ; p=0.478)$, concomitant asthma $(p=0.488, p=0.764)$. The G/G genotype in IL-17A (rs2275913) occurrence with coexisting 2282del4 FLG gene mutation increased the AD frequency 9 times $(p=0.0266)$.

Conclusions: The SNPs of IL-17A rs2275913 and IL-19 rs22431188 SNP seem not to have influence on AD course and occurrence while studied alone. The coexistence of GG genotype of IL-17A and 2282del4 FLG mutation may play a role as prognostic AD factor.
\end{abstract}

Key words: atopic dermatitis, filaggrin, IL-17A, IL-19, SNP.

\section{Introduction}

Atopic dermatitis (AD) is a chronic, recurrent, heterogeneous and inflammatory skin disease, which involves severe pruritus. The number of people suffering from AD is relatively high. Epidemiological research demonstrates that $20 \%$ of children and $2-5 \%$ of adults suffer from AD [1-3]. Atopic dermatitis pathogenesis is multifactorial and consists of genetic, immunological, and environmental factors with a skin barrier defect and microbial dysbiosis. A fresh look on the AD pathophysiology has focused on the skin defect barrier and immune dysfunctions.

Skin barrier defect includes disorders of cornified envelope proteins, intracellular lipids of stratum corneum abnormalities, antimicrobial peptides, tight junctions and proteases dysfunctions. Filaggrin is a major structural protein in the stratum corneum of the epidermis. The FLG gene is located in the epidermal differentiation complex on chromosome 1q21. The null mutations of the FLG gene observed in up to $50 \%$ of $A D$ patients are known as key players in $A D$ pathogenesis associated with the severe AD course, early onset, elevated IgE and concomitant asthma [4-6]. Skin barrier defect facilitates allergen and microbiome penetration and increases inflammation.

It is known that immune aberrations in AD concern Th2 lymphocyte disorders and the cytokines released by them, interleukin (IL)-4, IL-5, IL-13, leading to increased IgE processing, exacerbation of the skin inflammation and aggravation of the epidermal barrier defect [7]. Apart from the Th2-dependent response, lymphocytes Th17 and Th22 affect the inflammation of the skin in patients with atopic dermatitis releasing, among others, such cytokines as IL-17, IL-19, and IL-22 [8, 9]. It is widely known that Thelper 2 (Th2) and Th22 cells activation is the principal initiator of acute AD pathology, with the emergence of Th1 and Th17/IL-23 pathway activation marking the transition to a chronic state [10]. Ethnic differences in immunologic characteristics are observed.

Address for correspondence: Magdalena Trzeciak PhD, Department of Dermatology, Venereology and Allergology, Medical University of Gdansk, 17 Smoluchowskiego St, 80-214 Gdansk, Poland, phone: +48 585844010, e-mail: mtrzeciak@gumed.edu.pl Received: 20.11. 2020, accepted: 27.12.2020. 
The IL-17 family of cytokines is a group of homologous proteins: IL-17A and IL-17B to IL-17F [11]. Interleukin-17A, a distinctive cytokine, is produced not only by the T helper 17 cells (Th17 cells) but also by other cells such as cytotoxic CD8+ T cells (Tc17 cells), invariant natural killer T cells (iNKT cells), innate lymphoid cells (ILC), B cells, neutrophils, and other non-hematopoietic cells. All of these lymphocytes express the retinoic acid receptor-related orphan nuclear receptor C (RORC). During development, Th17 cells show flexible or plastic features distinct from those of Th1 and Th2 cells [12]. Genes for IL-17A are located on chromosome 6p12 [13]. Interleukin17A is a secreted pro-inflammatory cytokine that acts on/ affects a variety of cells, e.g. fibroblasts, epithelial cells, endothelial cells, and monocytes to induce the production of other cytokines, including IL-6, tumour necrosis factor- $\alpha$ (TNF- $\alpha)$, granulocyte-macrophage colony-stimulating-factor (GMCSF), granulocyte colony-stimulatingfactor (GCSF), chemokines (CXCL1, CXCL2, CXCL5, and CXCL8), antimicrobial peptides (defensins) and matrix metalloproteinases (MMP1, MMP3, and MMP13) [14, 15]. The effect of this process is the recruitment, activation and migration of neutrophils and myeloid cells, and also angiogenesis and tissue destruction.

Receptor A IL-17 (IL-17RA) belongs to the IL-17 family of receptors, which consists of five members (IL-17RA, IL-17RB, IL-17RC, IL-RD, IL-RE). They share sequence homology but IL-17RA is ubiquitously expressed on a wide range of tissues and cell types. Receptor IL-17 RA after stimulating IL-17 initiates and activates downstream signalling pathways of pro-inflammatory molecules production. However, IL-17RA stimulation is insufficient, because IL-17 signals must be transmitted by a heterodimeric receptor complex composed of IL-17RA and IL-17RC [16-18]. The IL-17A may affect the pathology of AD by different means. The murine model of AD lacks IL-17 attenuated skin inflammation, IgE and IL-4 production [19].

Furthermore, IL-17A induced the differentiation of Th2 producing IL-4, confirming that IL-17 also modulates Th2 mediated immune response [19]. Interleukin-17A exacerbates the phases of $A D$ by producing the downstream chemokines such as CXCL1, CXCL2, CXCL10, and CCL2 through the inhibition of EGFR (epidermal growth factor receptor) signalling pathway [20]. In the acute phase of $A D$, the level of IgE is increased. When cells were stimulated by IL-17A, B cells show increased survival, proliferation, and differentiation into plasma cells [21]. Research has confirmed that IL-17A provoked the IgE production by $B$ cells in humans [22].

Interleukin-17A enhances epidermal TJ barrier integrity [23]. Tight junctions are transmembrane proteins important in keratinocyte adhesion. Atopic dermatitis patients demonstrate proteins of tight junctions, particularly in filaggrin mutations [24].

Interleukin-19 (IL-19) is a pro-inflammatory cytokine that stimulates the production of type 2 T-helper cells (Th2)
[25]. Interleukin-19 belongs to the IL-10 family of cytokines (including IL-20, 22, 24, and 26). Its gene is located on chromosome 1q32 [26]. The human IL-19 gene is composed of seven exons and six introns and is encoded by exons 3-7. The IL-19 subfamily has 3 members, IL-19, IL-20 and IL-24 of a similar structure. The IL-19 receptor consists of IL-20R1 and IL-20R2. A heterodimer complex mediates its signal transduction and is an activator of transcription (STAT) 3 $[27,28]$. Vital cellular sources of IL-19 are monocytes [29] and keratinocytes [30]. In monocytes, lipopolysaccharide (LPS) and granulocyte macrophage colony stimulating factor (GM-CSF) upregulate IL-19 expression. Interleukin-19 stimulates IL-6 and tumour necrosis factor (TNF) expression and activates monocyte apoptosis and the production of reactive oxygen species (ROS) [31]. Activities and functions of IL-19 and IL-10 are different. IL-10 inhibits production of TNF- $\alpha$, IL-6, cell apoptosis, and ROS production, in contrast to IL-19. Monocytes may use the production of TNF- $\alpha$ to cause apoptosis in other cells as a defence mechanism against infection after receiving a signal from IL-19, which means a paracrine and not an autocrine effect. In turn, keratinocytes express IL-19 receptors suggesting that IL-19 may act in an autocrine manner [32]. Still little is known about the molecular mechanism of IL-19 signalling in the pathogenesis of AD; IL-19 which was originally associated with the development of type 2 helper T cell-responses and it is also associated with the development of type 1 helper T cell-responses [33]. It has been proved that IL-19 increases Th2 cytokine expression in activated T cells [34]. Oka et al. demonstrated that IL-19 protein levels in sera of $A D$ patients were significantly elevated compared with normal controls. Serum IL-19 levels increased according to AD severity. Konrad et al. demonstrated that in atopic dermatitis, IL-19 was significantly elevated, correlated with EASI scores, and lowered with skin improvement [35]. In vitro, IL-19 is strongly expressed in AD lesional skin under the influence of IL-17A, because it is up-regulated in keratinocytes by IL-17A and IL-4. It indicates that IL-19 may be important for bridging Th17 to Th2 in AD [25].

\section{Aim}

In our paper we have decided to examine the influence of the occurrence of polymorphism rs2275913 in the promoter region of the IL-17A gene and rs22431188 in the promoter region of IL-19 with $\mathrm{AD}$ alone and while combining 2282del4 FLG gene mutations. Concerning the complex character of $A D$, searching for prognostic panels in $A D$ is needed.

\section{Material and methods Material}

239 AD patients were analysed (140 women and 99 men) who were the patients of the Department of 
Dermatology, Venerology and Allergology, Medical University of Gdansk. Patients were recognised as suffering from $A D$ according to valid criteria proposed by Hanifin and Rajka [36]. The AD patients taking immunosuppressive treatment or other immunotherapy (UV phototherapy, cyclosporine A, oral corticoid, breast feeding and pregnant women, patients with inflammatory autoimmune and cancer diseases were excluded from the study. The control group was composed of 170 healthy persons (83 women and 87 men) with a negative individual and family interview for atopic illnesses. The groups were not ethnically different.

\section{Methods}

This study was performed to assess a potential association of AD with IL-17A, IL-19 genes and 2282del4 and R510X FLG mutation single nucleotide polymorphisms (SNPs). The analysis of polymorphic variants of IL-17A gene rs22431188 and IL-19 gene rs2275913 and 2282del4 and R510X FLG mutations was performed by the amplification - the polymerase chain reaction method (ARMS-PCR) using designed sequence-specific primers (PCR-SSP). All test samples were evaluated together with the growth hormone internal amplification control 1 (GH1). Genomic DNA was isolated from peripheral blood samples using Blood DNA Prep Plus (A\&A Biotechnology, Gdansk, Poland). Serum total lgE levels were measured by the fluoroimmunoenzyme assay using the Uni-CAP 100 System (Phadia, Uppsala, Sweden). The cut-off point for serum IgE was $100 \mathrm{kU} / \mathrm{l}$. All analyses were performed according to the manufacturer's protocols. Atopic dermatitis severity was assessed using the SCORAD scale (severe $(S C O R A D>50$, moderate (SCORAD 25-50, and mild (SCORAD < 25) [37]. The assessment of pruritus severity was performed using the visual analogue scale (VAS) (< 3 mild pruritus, 3-6.9- moderate pruritus, 7-8.9 - severe and 9-10 - very severe) [38].

The study was approved by the local ethics committee of the Medical University of Gdansk (no. NKBBN/ 113/2019).

\section{Statistical analysis}

Data were calculated for measurable variables: arithmetic mean $(\bar{x})$ and median $(\mathrm{Me})$ and standard deviation (SD), coefficient of variation ( $\mathrm{v} \%$ ). Minimum (min) and maximum ( $\max$ ) values were also given. Before making a comparison of averages in the examined groups, the coherence of analysed measurable variables with normal division was checked by means of Shapiro-Wilk test. When comparing averages in two groups, Mann-Whitney test was used, and in a few subgroups - ANOVA KruskalWallis test. In order to compare frequencies, different types of variables in examined groups and subgroups were used. To check the reliance between qualitative variables, unreliance test $\chi^{2}$ was performed. In the case of a theoretical numerical amount lower than 5, Yates alteration was taken into consideration. In case of a very small numerical amount in the chart $(<3)$, the Fisher's precise test was used. The odds ratio (OR) was calculated and reliance divisions were determined for them. The statistical significance of all tests is 0.05. Statistical analysis was made by means of statistical programme Statistica 10.0 StatSoft ${ }^{\circledR}[39]$.

\section{Results}

\section{Atopic dermatitis features}

The average age of AD patients was 25.5 years. The average age of controls was 27.1 years. Severity of AD was as follows: severe (SCORAD > 50, $n=66$ ), moderate (SCORAD 25-50, $n=50$ ), and mild (SCORAD $<25, n=21$ ). The average SCORAD score was 52.0 (48.3-52.7) 95\% Cl. Assessment of pruritus severity revealed: mild pruritus $20.7 \%$, moderate pruritus $37.9 \%$, severe $30.8 \%$ and very severe $10.6 \%$. The average pruritus was 5.65 (5.09-6.21; $95 \% \mathrm{Cl})$.

\section{Genotyping for IL-17A gene polymorphism rs2275913 and IL-19 polymorphism rs22431188}

Distribution of the genotypes and alleles for IL-17A and IL-19 was consistent with HWE (Hardy-Weinberg equilibrium) in patient and control groups and it is presented in Table 1.

We found three genotypes for IL-17A (G/G, G/A and $A / A)$ and for IL-19 (C/C, C/A, A/A) in both groups.

The distribution frequency of polymorphisms and alleles did not show any statistical significance $(p>0.05)$ (Table 1).

We found no evidence of any effect of IL-17A and IL-19 on the occurrence of $A D$ in the Polish population $(p>0.05)$.

\section{The association between rs2275913/17a and rs22431188/IL-19 polymorphisms with IgE levels, SCORAD score, pruritus severity and coexisting asthma}

We found no association between the presence of the SNPs of rs2275913/17a and rs22431188/IL-19 and IgE levels $(p>0.05)\left(\right.$ rs2275913/17a: $\chi^{2}=0.693 ; p=0.707$ and rs22431188/IL-19: $\chi^{2}=1.074 ; p=0.584$, respectively (Figure $1 \mathrm{~A}, \mathrm{~B}$ ).

No evidence was found of a link between rs2275913/ $17 \mathrm{a}$ and rs22431188/IL-19 polymorphisms with the AD intensification severity measured by SCORAD: mild, moderate and severe ( $p>0.05)$ (Figure 1 A, B; rs2275913/17a: $\chi^{2}=0.682, p=0.954$; rs22431188/IL-19: $\chi^{2}=3.367$, $p=0.498$, respectively).

In both rs2275913/17a and rs22431188/IL-19 polymorphisms, no significant relationship in average pruritus results was revealed nor the frequency of occur- 
Table 1. The comparison of the frequency of polymorphisms

\begin{tabular}{|c|c|c|c|c|c|c|}
\hline \multirow[t]{2}{*}{ Genotypes } & \multicolumn{2}{|c|}{ Patients } & \multicolumn{2}{|c|}{ Controls } & \multirow{2}{*}{$\begin{array}{l}\text { OR }(95 \% \mathrm{Cl}) \\
\text { Odds ratio }\end{array}$} & \multirow{2}{*}{$\begin{array}{l}\text { Statistical } \\
\text { significance }\end{array}$} \\
\hline & $n$ & $\%$ & $n$ & $\%$ & & \\
\hline \multicolumn{7}{|l|}{ IL-17A: } \\
\hline GG & 115 & 51.8 & 78 & 53.4 & Ref. & \multirow{3}{*}{$\begin{array}{l}\chi^{2}=0.245 \\
p=0.885\end{array}$} \\
\hline GA & 83 & 37.4 & 51 & 34.9 & $1.10(0.70-1.73)$ & \\
\hline AA & 24 & 10.8 & 17 & 11.6 & $1.04(0.52-2.06)$ & \\
\hline \multicolumn{7}{|l|}{ Alleles: } \\
\hline $\mathrm{G}$ & 313 & 70.5 & 207 & 70.9 & Ref. & \multirow{2}{*}{$\begin{array}{l}\chi^{2}=0.013 \\
p=0.909\end{array}$} \\
\hline A & 131 & 29.5 & 85 & 29.1 & $1.02(0.74-1.41)$ & \\
\hline \multicolumn{7}{|l|}{ IL-19: } \\
\hline $\mathrm{CC}$ & 151 & 63.7 & 109 & 66.5 & Ref. & \multirow{3}{*}{$\begin{array}{l}\chi^{2}=1.224 \\
p=0.542\end{array}$} \\
\hline CA & 78 & 32.9 & 47 & 28.7 & $1.20(0.77-1.86)$ & \\
\hline $\mathrm{AA}$ & 8 & 3.4 & 8 & 4.9 & $1.39(0.51-3.82)$ & \\
\hline \multicolumn{7}{|l|}{ Alleles: } \\
\hline C & 380 & 80.2 & 265 & 80.8 & Ref. & \multirow{2}{*}{$\begin{array}{l}\chi^{2}=0.047 \\
p=0.828\end{array}$} \\
\hline A & 94 & 19.8 & 63 & 19.2 & $1.04(0.73-1.48)$ & \\
\hline \multicolumn{7}{|c|}{ FLG rs2282del4 mutation: } \\
\hline $\mathrm{F} 1 / \mathrm{F} 1$ & 75 & 70.8 & 31 & 29.2 & & \multirow{3}{*}{$\begin{array}{l}\chi^{2}=7.669 \\
p=0.0213\end{array}$} \\
\hline $\mathrm{F} 1 / \mathrm{F} 2$ & 26 & 96.3 & 1 & 3.7 & $10.7(1.18-69.9)$ & \\
\hline $\mathrm{F} 2 / \mathrm{F} 2$ & & & & & & \\
\hline
\end{tabular}

rence of mild, moderate, severe and very severe pruritus $(p>0.05)$ (Figure 1 A, B).

No significant statistical correlation between asthma and polymorphisms rs2275913/17a $\left(p>0.05 ; \chi^{2}=1.436\right.$ $p=0.488)$ and $\mathrm{rs} 22431188 / \mathrm{IL}-19\left(p>0.05 ; \chi^{2}=0.539\right.$ $p=0.764$ ) was found.

\section{Association between rs2275913/IL-17A and rs22431188/IL-19 polymorphisms and concomitant 2282del4 FLG mutation and the frequency of $A D$}

The connection of IL-17a-rs2275913/17a and rs22431188/IL-19 polymorphisms with 2282del4 FLG mutation gene was examined (Table 2).

There has been a significant statistical link of AD occurrence in patients with G/G IL-17A polymorphism with 2228del4 FLG mutation revealed $\left(p=0.0266, \chi^{2}=4.917\right)$. It turned out that in the group of patients with the $\mathrm{G} / \mathrm{G}$ polymorphism, the frequency of $A D$ was not statistically higher than in controls, however when we combine the G/G polymorphism and FLG mutation, such a combination was much more frequent among AD patients compared to the control group (92.3\% vs. $7.7 \%$; OR $=9.1$; confidence interval is 1.16-71.4). This means that among the study subjects having the polymorphism and FLG mutation, AD occurs 9 times more often than in the study subjects with the GG polymorphism only.
Similar statistical difference in the AD occurrence was noticed in the case of patients with the G/A polymorphism of IL-17a and FLG mutation. It turned out that in the group of the G/A genotype in IL-17a (rs2275913) the frequency of $A D$ was much smaller than in the group of patients with the G/A polymorphism and FLG mutation (0\% vs. $100.0 \% ; \chi^{2}=5.707 ; p=0.0169$ ).

In the group of the C/C IL-19 polymorphism, the frequency of $A D$ was much smaller than in the group of patients with the CC polymorphism and FLG mutation present. A significant statistical difference was noted (0\% vs. $100.0 \% ; p<0.01 ; \chi^{2}=10.490, p=0.0012$ ).

In turn, there has been a non-significant but close to statistical significance of AD occurrence revealed in patients with the C/A IL-19 polymorphism. It turned out that in the group of patients with the C/A polymorphism, the frequency of $A D$ was much lower than in the study group with the C/A polymorphism and FLG gene mutation $(10 \%$ and $90.0 \%$; OR $=6.0)$. The reliance division is $0.74-49.0$ ( $p=0.055)$. This means that among the study subjects having the C/A polymorphism and FLG mutation, AD occurs 6 times more often than in the study subjects with the polymorphism only. As it can be seen, among 10 patients with a FLG mutation and C/A IL-19 polymorphism, 9 suffered from $A D$ and one was from the control group.

The frequency of FLG rs2282del4 and R501X mutation was examined. A statistically significant correlation 

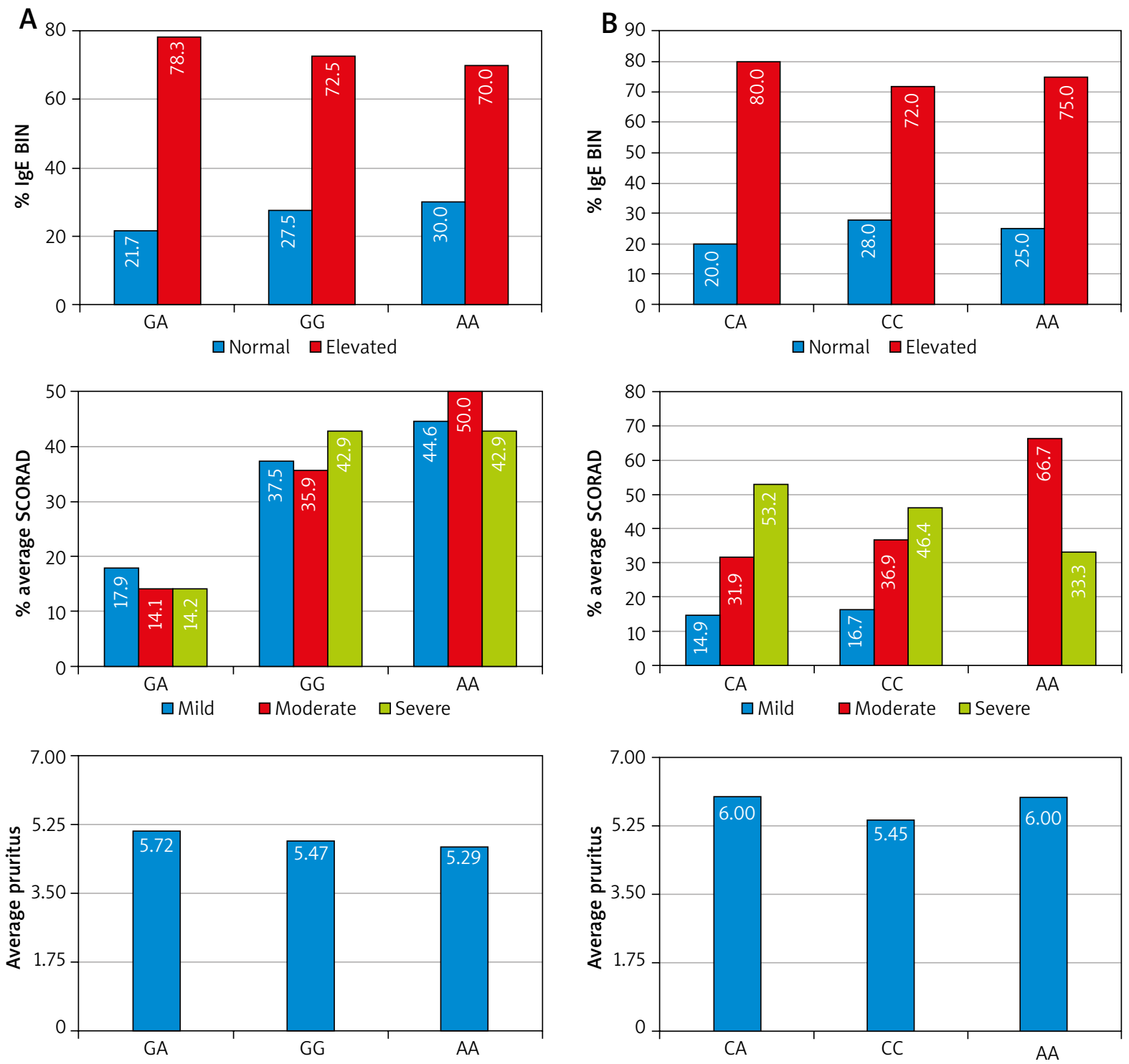

Figure 1. A - Average IgE BIN level (normal IgE $\leq 100$, elevated IgE $>100$ ), SCORAD score (mild, moderate and severe) and average pruritus severity in patients depending on IL-17A polymorphisms. B - Average IgE BIN level (normal IgE $\leq 100$, elevated IgE > 100), SCORAD score (mild, moderate and severe) and average pruritus severity in patients depending on IL-19 polymorphisms

was found in the results distribution for FLG rs2282del4 mutation in the patient and control group $(p<0.05$; $\left.\chi^{2}=7.699 ; p=0.0213, \mathrm{OR}=10.7 ; 95 \% \mathrm{Cl}: 1.18-69.9\right)$.

This section may be divided by subheadings. It should provide a concise and precise description and interpretation of the experimental results as well as the experimental conclusions that can be drawn.

\section{Discussion}

Atopic dermatitis often coexists with other atopic diseases such as conjunctivitis, allergic rhinitis or asthma. It is a chronic, recurrent, heterogeneous and inflammatory skin disease, which involves severe pruritus. Atopic dermatitis pathogenesis is multifactorial and consists of genetic, immunological and environmental factors with the skin barrier defect and microbial dysbiosis.

Some part of AD vulnerability was identified. The FLG mutation is a risk factor for $A D$ in the European population. It exists in one tenth of the Northern European population but only $1 \%$ of Africans $[40,41]$. Our results confirm a significant FLG mutation contribution to $A D$ pathogenesis.

Traditionally, it is believed that Th cells differentiate into Th1 and Th2 subgroups. Atopic dermatitis is characterized by a strong Th2-dependent immune response. However, lymphocytes Th1, Th22, Th17/IL-23 have an influence on the inflammation process. IL-17 is produced 
Table 2. Comparison of the frequency of separate polymorphisms and alleles in the patients and control groups for IL-17A and IL-19

\begin{tabular}{|c|c|c|c|c|c|c|}
\hline Group & $n$ & $\%$ & $n$ & $\%$ & $\begin{array}{l}\text { Odds ratio } \\
\text { OR }(95 \% \mathrm{Cl})\end{array}$ & $\begin{array}{c}\text { Statistical } \\
\text { significance }\end{array}$ \\
\hline \multicolumn{7}{|c|}{ Polymorphism IL-17A } \\
\hline & \multicolumn{2}{|c|}{ Genotype G/G } & \multicolumn{2}{|c|}{$\begin{array}{c}\text { Genotype G/G } \\
\text { +2282del4 FLG mutation }\end{array}$} & & \\
\hline Patients & 103 & 56.9 & 12 & 92.3 & \multirow[t]{2}{*}{$9.1(0.70-1.73)$} & \multirow{2}{*}{$\begin{array}{l}\chi^{2}=4.917 \\
p=0.0266\end{array}$} \\
\hline Controls & 78 & 43.1 & 1 & 7.7 & & \\
\hline Total & 181 & 100 & 13 & 100 & & \\
\hline \multicolumn{3}{|c|}{ Genotype G/A } & \multicolumn{2}{|c|}{$\begin{array}{c}\text { Genotype G/A } \\
\text { +2282del4 FLG mutation }\end{array}$} & & \\
\hline Patients & 72 & 58.5 & 11 & 100.0 & - & \multirow{2}{*}{$\begin{array}{l}\chi^{2}=5.707 \\
p=0.0169\end{array}$} \\
\hline Controls & 51 & 41.5 & - & - & - & \\
\hline Total & 123 & 100 & 11 & 100 & & \\
\hline \multicolumn{3}{|c|}{ Genotype A/A } & \multicolumn{2}{|c|}{$\begin{array}{c}\text { Genotype A/A } \\
\text { +2282del4 FLG mutation }\end{array}$} & & \\
\hline Patients & 22 & 56.4 & 2 & 100.0 & - & \multirow[t]{2}{*}{$p=0.337$} \\
\hline Controls & 17 & 43.6 & - & - & - & \\
\hline Total & 39 & 100 & 2 & 100 & & \\
\hline \multicolumn{7}{|c|}{ Polymorphism IL-19 } \\
\hline \multicolumn{3}{|c|}{ Genotype C/C } & \multicolumn{2}{|c|}{$\begin{array}{c}\text { Genotype C/C } \\
+ \text { 2282del4 FLG mutation }\end{array}$} & & \\
\hline Patients & 135 & 55.3 & 16 & 100.0 & - & \multirow{2}{*}{$\begin{array}{l}\chi^{2}=10.490 \\
p=0.0012\end{array}$} \\
\hline Controls & 109 & 44.7 & - & - & - & \\
\hline Total & 244 & 100 & 16 & 100 & & \\
\hline \multicolumn{3}{|c|}{ Genotype C/A } & \multicolumn{2}{|c|}{$\begin{array}{c}\text { Genotype C/A } \\
+2282 \text { del4 FLG mutation }\end{array}$} & & \\
\hline Patients & 69 & 60.0 & 9 & 90.0 & \multirow{2}{*}{$6.0(0.74-49.0)$} & \multirow{2}{*}{$p=0.055$} \\
\hline Controls & 46 & 40.0 & 1 & 10.0 & & \\
\hline Total & 115 & 100 & 10 & 100 & & \\
\hline \multicolumn{3}{|c|}{ Genotype A/A } & \multicolumn{2}{|c|}{$\begin{array}{c}\text { Genotype A/A } \\
+ \text { 2282del4 FLG mutation }\end{array}$} & & \\
\hline Patients & 7 & 46.6 & 1 & 100.0 & - & \multirow[t]{2}{*}{$p=0.50$} \\
\hline Controls & 8 & 53.4 & - & - & - & \\
\hline Total & 15 & 100 & 1 & 100 & & \\
\hline
\end{tabular}

by subgroups of T CD4 + lymphocytes - Th17 cells. The Th17 cells are vital to the adaptive immune response against bacterial and fungal infections, and also contribute to the pathogenesis of several inflammatory diseases.

The pathogenetic role of Th17 and IL-17 cells in different autoimmune and inflammatory processes was observed [42-44]. IL-17A which is produced by type 17 helper $T$ cells has been related to neutrophilic inflammation in asthma [45]. Thus, numerous research in different populations was aimed to examine the connection between the IL-17A polymorphism not only with AD, but also with asthma. In Portuguese adults, a connec- tion between polymorphisms of the IL-17A (rs2275913) gene with rhinitis and/or asthma was evaluated. IL-17A rs2275913 AA genotype was less associated with asthma than with rhinitis [43].

The research conducted in Saudi Arabia showed that AG heterozygotes of rs17880588 in IL-17A were significantly more common in the control group than among asthma patients. For any of the other SNPs examined (rs17878530), no significant associations were observed in this population. Hence, the results revealed an association between rs17880588 (G/A) and protection from asthma in the Saudi population [46]. Research results in the Chinese population preliminarily indicate that the 
IL-17A genotype AT (rs7758579 - rs11966760) was thought to be a risk of allergy rhinitis (AR) - asthma (A). Moreover, SNPs and some intergenic variants have a potential association with AR and comorbid asthma [47]. Maalmi results suggested that SNPS in IL-17A and IL-17F confer susceptibility to childhood asthma in Tunisia [48]. However, Wang et al. failed to find an association between IL-17A rs2275913 polymorphism and asthma risk [49]. The variant genotype of IL-17A rs2275913 polymorphism GA or AA was a significant protective factor for asthma in schoolchildren aged 7 to 13 years in the study group with bronchiolitis in early infancy [50].

In the meta-analysis assessing the effect of IL-17A rs2275913 polymorphism on the risk of asthma, ten studies with a total of 5016 participants (2510 asthma cases and 2506 controls) were ultimately identified. The results of this meta-analysis indicate that the $\mathrm{G}$ allele of rs2275913 in IL-17A is a protective factor for the development of asthma. The G allele of rs2275913 in IL-17A is significantly associated with a reduced risk of asthma in children and Asians, in subgroup analysis by age and ethnicity [51].

Narbutt et al. found that the A/A genotype in IL-17A predisposed to increased $A D$ severity and coexistence of $A D$ and asthma [52]. However, they found no evidence of any effect of IL-17A on the occurrence of $A D$ in the Polish population, which was consistent with our results.

However, we found no correlation between the AD severity and asthma coexistence combined with examined polymorphisms IL-17A. This may be a result of different ethnic roots in these two parts of Poland. In our study, patients were mainly from northern Poland rather than central Poland and the number of AD patients examined in our group was bigger.

Interleukin-19 is primarily made by monocytes, where its expression can be induced by LPS, IL-4, and GM-CSF. IL-19 signals through a receptor complex composed of the IL-20R1 as well as IL-20R2 chains and activates monocytes in an autocrine and paracrine way to release cytokines IL-6, TNF- $\alpha$, and numerous ROSs. Interleukin-19 stimulates the production of Type 2 T-helper cells (Th2). It is thought that IL-19 is crucial for bridging Th17 to Th2 pathway.

What is also important, they put attention to the possible influence of FLG mutation coexisting with certain IL-17 and IL-19 polymorphisms on the AD occurrence. The combination of FLG mutations and SNPS of IL-17A and IL-19 influences the frequency of AD occurrence.

Expression of epidermal FLG is down regulated by cytokines IL-4, IL-13, IL-17A, IL-22, IL-25, IL-31, TNF- $\alpha$ in AD patients [53-59].

Filaggrin expression at the mRNA level is directly and indirectly downregulated by IL-17A by affecting profilaggrin mRNA expression, production of functional filaggrin monomers and their degradation at the level of enzymatic processing. Keratinocytes stimulation with IL-17A alters many cellular processes that can affect the barrier func- tion of the skin, with a special influence on the response to inflammation, epidermal differentiation, formation of cornified envelope, and stress as well as chemotaxis [54]. It is also worth reminding the study by Teunissen et al. which reported that IL-17A upregulates both HLA-DR molecules and ICAM-1 in keratinocytes. It contributes to the formation of a functional immunological synapse and enhancement of antigen presenting function of these cells [60].

Research on polymorphism genes of IL-19 is not common. In the north Indian population, IL-19-35402 C/C (rs2243158) genotype was associated with an increased risk of psoriasis. Researchers did not find any significant association of IL-19-42232 C/A (rs2243188), -38386 T/C (rs2073186) with psoriasis [61]. In turn, the study on the European population reveals that there is no significant difference of IL-19 gene SNPs in psoriasis patients and controls [62]. Interleukin-19 belongs to the IL-10 family of cytokines and its gene is located on chromosome 1 q32. Interleukin-10-1082 A/G, -819 T/C and -592 A/C polymorphisms were not strongly connected with the susceptibility to atopic dermatitis [63].

There might be a phenomenon existing in genetics of the additive influence of coexistence of 2282del4 FLG mutations with the polymorphism in promotor region rs2275913 of IL-17A and rs22431188 of IL-19 gene SNPS on the development of AD. It is claimed that patients with a 2282del4 FLG mutation who have certain polymorphism variants are at a greater risk of an illness occurrence than patients with polymorphisms only and without FLG mutation.

Limitations of the study may result from the fact that the group was ethnically limited and there could be some gene-environment interaction. Patients were heterogeneous as far as AD severity and its course are concerned. The majority had a moderate and severe course, which may influence the results. Moreover, the number of patients taking part in the research was also limited.

These discrepancies could be due to differences in patient demographics, sample size, environmental factors, and genetic background, all of which can affect association studies.

Various research results may come from choosing a different group of patients so further cytokines research is vital. Our research results, on the contrary, showed the correlation of AD occurrence in the case of polymorphisms IL-17A and IL-19 with coexisting FLG mutation. It may indicate an additive influence of immune and genetic disorders on AD pathogenesis. We are aware that both IL-17A and IL-19 play an essential role in pathophysiology of AD.

Research continuation in the future with even better patient choice will not only provide better understanding of the AD mechanism but also contribute to the therapy individualization. It is a vital direction in the future research. 


\section{Conclusions}

Our research gives new insights into the complex AD pathophysiology on mutual interaction of 2282del4 FLG mutations and SNPs of IL-17A (rs2275913) and IL-19 (rs22431188) which have an influence on AD occurrence. It seems that in this complex disease, not only one genetic marker, but rather panels are useful for diagnostics and prediction.

\section{Acknowledgments}

Grant no. 02-0066/07 has been received from the Polish Ministry of Science and Higher Education.

\section{Conflict of interest}

The authors declare no conflict of interest.

\section{References}

1. Mortz CG, Andersen KE, Dellgren C, et al. Atopic dermatitis from adolescence to adulthood in the TOACS cohort: prevalence, persistence and comorbidities. Allergy 2015; 70: 83645.

2. Barbarot S, Auziere S, Gadkari A, et al. Epidemiology of atopic dermatitis in adults: results from an international survey. Allergy 2018; 73: 1284-93.

3. Asher MI, Montefort S, Björkstén B, et al. Worldwide time trends in the prevalence of symptoms of asthma, allergic rhinoconjunctivitis, and eczema in childhood: ISAAC Phases One and Three repeat multicountry cross-sectional surveys. Lancet 2006; 368: 733-43.

4. Palmer CN, Irvine AD, Terron-Kwiatkowski A, et al. Common loss-of-function variants of the epidermal barrier protein filaggrin are a major predisposing factor for atopic dermatitis. Nat Genet 2006; 38: 441-6.

5. van den Oord RA, Sheikh A. Filaggrin gene defects and risk of developing allergic sensitisation and allergic disorders: systematic review and meta-analysis. BMJ 2009; 339: b2433.

6. Rodríguez E, Baurecht $\mathrm{H}$, Herberich E, et al. Meta-analysis of filaggrin polymorphisms in eczema and asthma: robust risk factors in atopic disease. J Allergy Clin Immunol 2009; 123: 1361-70.e7.

7. Guttman-Yassky E, Krueger JG. Atopic dermatitis and psoriasis: two different immune diseases or one spectrum? Curr Opin Immunol 2017; 48: 68-73.

8. Gittler JK, Shemer A, Suárez-Farińas M, et al. Progressive activation of $T(H) 2 / T(H) 22$ cytokines and selective epidermal proteins characterizes acute and chronic atopic dermatitis. J Allergy Clin Immunol 2012; 130: 1344-54.

9. Noda S, Suárez-Farińas M, Ungar B, et al. The Asian atopic dermatitis phenotype combines features of atopic dermatitis and psoriasis with increased TH17 polarization. J Allergy Clin Immunol 2015; 136: 1254-64.

10. Guttman-Yassky E, Krueger JG, Lebwohl MG. Systemic immune mechanisms in atopic dermatitis and psoriasis with implications for treatment. Exp Dermatol 2018; 27: 409-17.

11. Moseley TA, Haudenschild DR, Rose L, Reddi AH. Interleukin-17 family and IL-17 receptors. Cytokine Growth Factor Rev 2003; 14: 155-74.

12. Cua DJ, Tato CM. Innate IL-17-producing cells: the sentinels of the immune system. Nat Rev Immunol 2010; 10: 479-89.
13. Atlas of Genetics and Cytogenetics in Oncology and Haematology. Available at: http://atlasgeneticsoncology.org/Genes/ GC IL17A.html (Accessed: 12.09.2020).

14. Eyerich S, Eyerich K, Cavani A, Schmidt-Weber C. IL-17 and IL-22: siblings, not twins. Trends Immunol 2010; 31: 354-61.

15. Iwakura Y, Ishigame H, Saijo S, Nakae S. Functional specialization of interleukin-17 family members. Immunity 2011; 34: 149-62.

16. Toy D, Kugler D, Wolfson M, et al. Cutting edge: interleukin 17 signals through a heteromeric receptor complex. I Immunol 2006; 177: 36-9.

17. Song X, Zhu S, Shi P, et al. IL-17RE is the functional receptor for IL-17C and mediates mucosal immunity to infection with intestinal pathogens. Nat Immunol 2011; 12: 1151-58.

18. Rickel EA, Siegel LA, Yoon BR, et al. Identification of functional roles for both IL-17RB and IL-17RA in mediating IL-25-induced activities. J Immunol 2008; 181: 4299-310.

19. Nakajima S, Kitoh A, Egawa G, et al. IL-17A as an inducer for Th2 immune responses in murine atopic dermatitis models. J Invest Dermatol 2014; 134: 2122-30.

20.Zhang Z, Xiao C, Gibson AM, et al. EGFR signaling blunts allergen-induced IL- 6 production and Th17 responses in the skin and attenuates development and relapse of atopic dermatitis. J Immunol 2014; 192: 859-66.

21. Doreau A, Belot A, Bastid J, et al. Interleukin 17 acts in synergy with $B$ cell-activating factor to influence $B$ cell biology and the pathophysiology of systemic lupus erythematosus. Nat Immunol 2009; 10: 778-85. [Retracted in: Nat Immunol 2014; 15: 894

22. Milovanovic M, Drozdenko G, Weise C, et al. Interleukin-17A promotes IgE production in human B cells. J Invest Dermatol 2010; 130: 2621-8.

23. Brewer MG, Yoshida T, Kuo Fl, et al. Antagonistic effects of IL-4 on IL-17A-mediated enhancement of epidermal tight junction function. Int J Mol Sci 2019; 20: 4070.

24. Gruber R, Elias PM, Crumrine D, et al. Filaggrin genotype in ichthyosis vulgaris predicts abnormalities in epidermal structure and function. Am J Pathol 2011; 178: 2252-63.

25. Oka T, Sugaya M, Takahashi N, et al. Increased Interleukin-19 expression in cutaneous T-cell lymphoma and atopic dermatitis. Acta Derm Venereol 2017; 97: 1172-7.

26. Dembic Z. The cytokines of the immune system. The role of cytokines in disease related to immune response. $1^{\text {st }}$ ed. Academic Press, USA 2015; 99-122.

27. Blumberg H, Conklin D, Xu WF, et al. Interleukin 20: discovery, receptor identification, and role in epidermal function. Cell 2001; 104: 9-19.

28. Dumoutier L, Leemans C, Lejeune D, et al. Cutting edge: STAT activation by IL-19, IL-20 and mda-7 through IL-20 receptor complexes of two types. J Immunol 2001; 167: 3545-9.

29. Gallagher G, Dickensheets H, Eskdale J, et al. Cloning, expression and initial characterisation of interleukin-19 (IL-19), a novel homologue of human interleukin-10 (IL-10). Genes Immun 2000; 1: 442-50.

30. Kunz S, Wolk K, Witte E, et al. Interleukin (IL)-19, IL-20 and IL24 are produced by and act on keratinocytes and are distinct from classical ILs. Exp Dermatol 2006; 15: 991-1004.

31. Liao YC, Liang WG, Chen FW, et al. IL-19 induces production of IL-6 and TNF-alpha and results in cell apoptosis through TNF-alpha. J Immunol 2002; 169: 4288-97.

32. Witte $\mathrm{E}$, Kokolakis G, Witte K, et al. IL-19 is a component of the pathogenetic IL-23/IL-17 cascade in psoriasis. I Invest Dermatol 2014; 134: 2757-67. 
33. Fujimoto Y, Aono K, Azuma YT. The clarified role of interleukin-19 in the inflammatory bowel disease and hypersensitivity: insights from animal models and humans. J Vet Med Sci 2019; 81: 1067-73.

34. Gallagher G, Eskdale J, Jordan W, et al. Human interleukin-19 and its receptor: a potential role in the induction of Th2 responses. Int Immunopharmacol 2004; 4: 615-26.

35. Konrad RJ, Higgs RE, Rodgers GH, et al. Assessment and clinical relevance of serum IL-19 levels in psoriasis and atopic dermatitis using a sensitive and specific novel immunoassay. Sci Rep 2019; 9: 5211.

36. Hanifin JM, Rajka G. Diagnostic features of atopic dermatitis Acta Derm Venereol Suppl 1980; 92: 44-7.

37. Wollenberg A, Christen-Zäch S, Taieb A, et al. ETFAD/EADV Eczema task force 2020 position paper on diagnosis and treatment of atopic dermatitis in adults and children. J Eur Acad Dermatol Venereol 2020; 34: 2717-44.

38. Wollenberg A, Barbarot S, Bieber T, et al. Consensus-based European guidelines for treatment of atopic eczema (atopic dermatitis) in adults and children: part II. J Eur Acad Dermatol Venereol 2018; 32: 850-78.

39. Stanisz A. Przystępny kurs statystyki z zastosowaniem STATISTICA PL na przykładach z medycyny Tom 1. Statystyki podstawowe. Kraków: StatSoft; 2006.

40. Thawer-Esmail F, Jakasa I, Todd G, et al. South African amaXhosa patients with atopic dermatitis have decreased levels of filaggrin breakdown products but no loss-of-function mu tations in filaggrin. J Allergy Clin Immunol 2014; 133: 280-2. e22.

41. Drislane C, Irvine AD. The role of filaggrin in atopic dermatitis and allergic disease. Ann Allergy Asthma Immunol 2020; 124: 36-43.

42. Eskandari-Nasab E, Moghadampour M, Tahmasebi A. Metaanalysis of risk association between interleukin-17A and $F$ gene polymorphisms and inflammatory diseases. J Interferon Cytokine Res 2017; 37: 165-74.

43. Resende EP, Todo-Bom A, Loureiro C, et al. Asthma and rhinitis have different genetic profiles for IL13, IL17A and GSTP1 polymorphisms. Rev Port Pneumol (2006) 2017; 23: 10-6.

44.Espinoza JL, Takami A, Nakata K, et al. A genetic variant in the IL-17 promoter is functionally associated with acute graft-versus-host disease after unrelated bone marrow transplantation. PLoS One 2011; 6: e26229.

45. Bullens DM, Truyen E, Coteur L, et al. IL-17 mRNA in sputum of asthmatic patients: linking $T$ cell driven inflammation and granulocytic influx? Respir Res 2006; 7: 135.

46. Bazzi MD, Sultan MA, Al Tassan N, et al. Interleukin 17A and $F$ and asthma in Saudi Arabia: gene polymorphisms and protein levels. J Investig Allergol Clin Immunol 2011; 21: 551-5.

47. Wang M, Zhang Y, Han D, Zhang L. Association between polymorphisms in cytokine genes IL-17A and IL-17F and development of allergic rhinitis and comorbid asthma in Chinese subjects. Hum Immunol 2012; 73: 647-53.

48. Maalmi H, Beraies A, Charad R, et al. IL-17A and IL-17F genes variants and susceptibility to childhood asthma in Tunisia. J Asthma 2014; 51: 348-54.

49. Wang J, Zhou J, Lin LH, et al. Association of single nucleotide polymorphism of IL-17 gene promoter with childhood asthma. Acad J Second Military Med Univ 2011; 32: 481-4.

50. Holster A, Teräsjärvi J, Lauhkonen E, et al. IL-17A gene polymorphism rs2275913 is associated with the development of asthma after bronchiolitis in infancy. Allergol Int 2018; 67: 109-13.
51. Zhai C, Li S, Feng W, et al. Association of interleukin-17a rs2275913 gene polymorphism and asthma risk: a metaanalysis. Arch Med Sci 2018; 14: 1204-11.

52. Narbutt J, Wojtczak M, Zalińska A, et al. The A/A genotype of an interleukin-17A polymorphism predisposes to increased severity of atopic dermatitis and coexistence with asthma. Clin Exp Dermatol 2015; 40: 11-6.

53. Howell MD, Kim BE, Gao P, et al. Cytokine modulation of atopic dermatitis filaggrin skin expression. J Allergy Clin Immunol 2009; 124 (3 Suppl 2): R7-12.

54.Gutowska-Owsiak D, Schaupp AL, Salimi M, et al. IL-17 downregulates filaggrin and affects keratinocyte expression of genes associated with cellular adhesion. Exp Dermatol 2012; 21: 104-10.

55. Cornelissen C, Marquardt Y, Czaja K, et al. IL-31 regulates differentiation and filaggrin expression in human organotypic skin models. J Allergy Clin Immunol 2012; 129: 426-33.e4338.

56.Deleuran M, Hvid M, Kemp K, et al. IL-25 induces both inflammation and skin barrier dysfunction in atopic dermatitis. Chem Immunol Allergy 2012; 96: 45-9.

57. Gutowska-Owsiak D, Schaupp AL, Salimi M, et al. Interleukin-22 downregulates filaggrin expression and affects expression of profilaggrin processing enzymes. Br J Dermatol 2011; 165: 492-8.

58. Hvid M, Johansen C, Deleuran B, et al. Regulation of caspase 14 expression in keratinocytes by inflammatory cytokines a possible link between reduced skin barrier function and inflammation? Exp Dermatol 2011; 20: 633-6.

59. Kim BE, Howell MD, Guttman-Yassky E, et al. TNF- $\alpha$ downregulates filaggrin and loricrin through $\mathrm{c}$-Jun $\mathrm{N}$-terminal kinase: role for TNF- $\alpha$ antagonists to improve skin barrier. J Invest Dermatol 2011; 131: 1272-9. Erratum in J Invest Dermatol 2011; 131: 1388

60. Teunissen MB, Koomen CW, de Waal Malefyt R, et al. Interleukin-17 and interferon-gamma synergize in the enhancement of proinflammatory cytokine production by human keratinocytes. J Invest Dermatol 1998; 111: 645-9.

61. Wani A, Bashir AG, Tahseena A, et al. Possible association of proinflammatory cytokine IL-19 gene polymorphism with psoriasis. Genet Mol Res 2019; 18; https://www.geneticsmr. org/articles/possible-association-of-proinflammatory-cytokine-il19-gene-polymorphism-with-psoriasis-7636.html.

62. Galimova E, Rätsep R, Traks T, et al. Interleukin-10 family cytokines pathway: genetic variants and psoriasis. Br J Dermatol 2017; 176: 1577-87.

63. Qi Y, Kong J, He J. Genetic relationship between IL-10 gene polymorphisms and the risk of clinical atopic dermatitis. BMC Med Genet 2019; 20: 83. 